\title{
Photometry and imaging of comet C/2004 Q2 (Machholz) at Lulin and La Silla
}

\author{
Z. Y. $\operatorname{Lin}^{1}$, M. Weiler ${ }^{2}$, H. Rauer ${ }^{2}$, and W. H. Ip ${ }^{1}$ \\ 1 Institute of Astronomy, National Central University, 300 Jungda Rd, Jungli City, Taiwan \\ e-mail: s1249002@cc.ncu.edu.tw \\ 2 Institut fur Planetenforschung, DLR, Rutherfordstr. 2, 12489 Berlin, Germany
}

Received 13 February 2007 / Accepted 20 March 2007

\section{ABSTRACT}

\begin{abstract}
Aims. We have investigated the development of the dust and gas coma of the comet C/2004 Q2 (Machholz) from December 2004 to January 2005 using observations obtained at the Lulin Observatory, Taiwan and the European Southern Observatory at La Silla, Chile. Methods. We determined the dust-activity parameter Af $\rho$ and the dust color and derived Haser production rates and scale lengths of $\mathrm{C}_{2}$ and $\mathrm{NH}_{2}$. An image enhancement technique was applied to study the morphology of the gas and dust coma.

Results. Two jets were observed in the coma distributions of $\mathrm{C}_{2}$ and $\mathrm{CN}$ of comet Machholz that were not formed in the dust coma. The jets showed a spiral-like structure and pointed at different position angles at different observing times. The formation of these jets can be explained by the presence of two active surface regions on a rotating cometary nucleus.
\end{abstract}

Key words. comets: indvidual: C/2004Q2 (Machholz)

\section{Introduction}

Comet C/2004 Q2 (Machholz), moving on an orbit with an eccentricity of 0.9995 and an inclination to the ecliptic plane of $38.6^{\circ}$, was discovered by Don Machholz on August 27, 2004 (IAUC 8394, 2004). The orbital period was approximately determined to be 113000 years (MPC 54558), thereby making comet Machholz a likely member of the distant Oort cloud population of primordial icy objects. Due to proximity to the Earth (0.35 AU) on January 5th, 2005, comet Machholz showed its maximum magnitude $(3 \sim 4)$ at the beginning of January and had become a naked-eye object for several weeks. Its perihelion passage, at a heliocentric distance of $1.21 \mathrm{AU}$, was on January 25, 2005. Sastri et al. (IAUC 8480, 2005) reported a fanlike structure containing up to three jets on the sunward side of cometary coma during early January 2005. From the evolution of these structures, the rotation period of the nucleus of comet Machholz was determined to be $0.38 \pm 0.08$ days.

In this paper, we describe narrow-band filter imaging observations of comet Machholz performed between early December 2004 and late January 2005. An overview of the observations is presented in Table 1 . The observations used narrowband filters centered on cometary gas emissions of $\mathrm{C}_{2}$ and $\mathrm{NH}_{2}$ and the continuum in two wavelength regions. In four nights of the observing programs at Lulin and LaSilla ${ }^{1}$, the sky conditions were photometric and the observations of those nights were used for determining the $A f \rho$ parameter and the gas production rates. The observations and their reduction are described in Sect. 2. In Sect. 3, we analyze the dust continuum and gas emissions, and in Sects. 4 and 5, we study the coma morphology and discuss the implications. In Sect. 6, we summarize our results.

${ }^{1}$ Partly based on obvervations collected at the European Southern Observatory, Chile (ESO program 274.C-5020).

\section{Observations and data reduction}

Two sets of narrow-band filter images are presented in this paper. The first set was obtained using the 1-m telescope of the Lulin Observatory, Taiwan, from December 1, 2004 to January 25, 2005. This time period included the comet's closest approach to Earth (2005 Jan. 5.61, 0.347 AU) and its perihelion passage (2005 Jan. 25.20, 1.21 AU). The observations were made with the ESA Rosetta comet filters (Bockelee-Morvan et al. 2004) centered on the emissions of $\mathrm{C}_{2}(\Delta v=0)$ and $\mathrm{NH}_{2}(0,7,0)$ and on two wavelength ranges containing solar continuum only ("Blue" continuum at $445 \mathrm{~nm}, F W H M=4 \mathrm{~nm}$ and the "Red" continuum at $687.4 \mathrm{~nm}, F W H M=6 \mathrm{~nm}$ ). For the Lulin observations, a PI1300B $1340 \times 1300$ CCD camera with an effective pixel scale of $0.515^{\prime \prime}$ pixel $^{-1}$ (Kinoshita et al. 2005) and an AP8 $1024 \times 1024$ CCD camera with a pixel scale of $0.62^{\prime \prime}$ pixel $^{-1}$ (Kinoshita et al. 2002) were used. The second set of observations was acquired with the $3.6 \mathrm{~m}$ telescope of ESO (European Southern Observatory) in La Silla, Chile, using the EFOSC2 instrument $\left(1030 \times 1030\right.$ pixels, pixel size: $0.314^{\prime \prime}$, FOV $\left.5.39^{\prime} \times 5.39^{\prime}\right)$. The observations employed filters centered on the gas emissions of $\mathrm{CN}(\Delta v=0)$ and $\mathrm{C}_{2}(\Delta v=0)$ and on two continuum regions (442.2 $\mathrm{nm}$ and $683.8 \mathrm{~nm}$ ). More details on the filter properties can be found in Lara et al. (2004).

The data reduction followed standard procedures. Briefly, the procedure began with bias and dark-current subtraction and flatfield correction of all frames. Then the night sky contribution was subtracted. For the observations obtained at the Lulin observatory in December 2004 the night sky level was determined from those parts of the comet images that do not contain contributions from the cometary coma. For the ESO observations in December 2004 and all observations during January 2005, separate sky background images, taken at a position about $0.5^{\circ}$ away from the comet's position in the sky, were used for the sky background determination. This procedure was necessary since the 
Table 1. Log of observations performed at the Lulin and La Silla observations.

\begin{tabular}{lcccccccc}
\hline \hline Date UT & $\begin{array}{c}r_{\mathrm{h}} \\
(\mathrm{AU})\end{array}$ & $\begin{array}{c}\Delta \\
(\mathrm{AU})\end{array}$ & $\begin{array}{c}\text { Solar } \\
\text { PA } \\
(\text { Degree })\end{array}$ & $\begin{array}{c}\text { Pixel } \\
\text { scale } \\
(\mathrm{km} / \text { pixel })\end{array}$ & $\Theta$ & Filters & CCD & Note \\
(Degree) & & & & \\
\hline Dec. 01 2004 & 1.47 & 0.614 & 173.6 & 229.30 & 30.4 & RF & PI1300B & \\
Dec. 05 2004 & 1.43 & 0.566 & 180.7 & 211.41 & 30.3 & RF & PI1300B & $\star$ \\
Dec. 09 2004 & 1.41 & 0.528 & 187.6 & 120.25 & 30.1 & ESO & PI1300B & \\
Dec. 15 2004 & 1.36 & 0.460 & 204.5 & 171.82 & 29.7 & RF & PI1300B & $\star$ \\
Dec. 29 2004 & 1.27 & 0.362 & 239.5 & 135.21 & 31.6 & RF & PI1300B & \\
Dec. 30 2004 & 1.27 & 0.358 & 242.6 & 133.70 & 32.1 & RF & PI1300B & \\
Jan. 06 2005 & 1.24 & 0.347 & 257.7 & 129.61 & 36.8 & RF & PI1300B & $\star$ \\
Jan. 24 2005 & 1.21 & 0.431 & 245.2 & 193.81 & 49.9 & RF & AP8 & $\star$ \\
Jan. 25 2005 & 1.21 & 0.441 & 244.7 & 198.31 & 50.4 & RF & AP8 & \\
\hline
\end{tabular}

Note: $\Delta$ and $r_{h}$ are the geocentric and heliocentric distances in AU; Solar PA is the position angle of the projected solar direction, measured from North towards East, and $\Theta$ is the phase angle, ie., the Sun-comet-observer angle. Filters "RF" means the Rosetta narrowband filters of $\mathrm{C}_{2}, \mathrm{NH}_{2}$, Blue cont. and Red cont. Filters "ESO" means images were taken from La Silla Observatory, ESO, by also using narrowband filters $\left(\mathrm{CN}, \mathrm{C}_{2}, \mathrm{BC}\right.$ and RC). Nights marked with $\star$ were photometric.

Table 2. Rosetta comet filter extinction coefficients from observations on four photometric nights.

\begin{tabular}{lcccc}
\hline \hline Date UT & $\begin{array}{c}\mathrm{BC} \\
(\mathrm{mag} / \mathrm{AM})\end{array}$ & $\begin{array}{c}\mathrm{RC} \\
(\mathrm{mag} / \mathrm{AM})\end{array}$ & $\begin{array}{c}\mathrm{C}_{2} \\
(\mathrm{mag} / \mathrm{AM})\end{array}$ & $\begin{array}{c}\mathrm{NH}_{2} \\
(\mathrm{mag} / \mathrm{AM})\end{array}$ \\
\hline Dec. 05 2004 & $0.165 \pm 0.008$ & $0.054 \pm 0.003$ & $0.080 \pm 0.001$ & - \\
Dec. 15 2004 & $0.220 \pm 0.009$ & $0.040 \pm 0.001$ & $0.118 \pm 0.001$ & - \\
Jan. 06 2005 & $0.180 \pm 0.010$ & $0.088 \pm 0.004$ & $0.141 \pm 0.006$ & $0.109 \pm 0.008$ \\
Jan. 24 2005 & $0.285 \pm 0.011$ & $0.074 \pm 0.008$ & $0.100 \pm 0.007$ & $0.094 \pm 0.008$ \\
\hline
\end{tabular}

Note: $\mathrm{BC}$ and RC mean "blue" continuum and "red" continuum filters, respectively, and $\mathrm{C}_{2}$ and $\mathrm{NH}_{2}$ mean filters centered on the $\mathrm{C}_{2}$ and $\mathrm{NH}_{2}$ emissions. In December 2004 no observations with the $\mathrm{NH}_{2}$ filter were performed.

Table 3. Af $\rho$ parameter determined from observations on four photometric nights.

\begin{tabular}{lccc}
\hline \hline Date(UT) & $\begin{array}{c}\mathrm{BC} \\
\text { Af } \rho_{445}(\mathrm{~cm})\end{array}$ & $\begin{array}{c}\mathrm{RC} \\
\text { Af } \rho_{687}(\mathrm{~cm})\end{array}$ & $\begin{array}{c}\text { Dust color } \\
(\% \text { per 1000 ̊) }\end{array}$ \\
\hline Dec. 05 2004 & $2683 \pm 224$ & $3198 \pm 66$ & $7.2 \pm 3.5$ \\
Dec. 15 2004 & $3139 \pm 275$ & $3187 \pm 32$ & $0.6 \pm 3.6$ \\
Jan. 06 2005 & $4032 \pm 113$ & $4216 \pm 53$ & $1.8 \pm 1.6$ \\
Jan. 24 2005 & $3128 \pm 125$ & $3892 \pm 53$ & $9.0 \pm 1.7$ \\
\hline
\end{tabular}

Note: The last column shows the color of the dust coma derived from the $A f \rho$ values at the two different wavelengths.

cometary coma filled the entire field-of-view of the instruments. The extinction coefficients for the observations at Lulin observatory were determined for all nights with photometric sky conditions, using at least three stars observed at a number of different airmasses during the night. The derived extinction coefficients of narrow-band filters are listed in Table 2. These coefficients were used for the extinction correction. Photometric standard stars, GD71 or BD+284211, selected from the list presented by Bonev et al. (2003) were observed on photometric nights and used to convert the measured counting rates into physical units. The continuum contribution in the gas-filter images was removed by subtraction of the appropriately scaled continuum determined from the dust filter images. The continuum scaling factor $(k)$ can be calculated by taking into account both the wavelength dependence of the solar spectrum and the effect of the reddened cometary continuum, as discussed later.

Note that in December 2004, no $\mathrm{NH}_{2}$ images were obtained at Lulin because the filter centered on the $\mathrm{NH}_{2}$ emission was replaced by a comet ion filter (i.e. $\mathrm{CO}^{+}$) for observing comet Machholz's ion tail. The observations of comet Machholz's ion tail will be described in a subsequent publication.

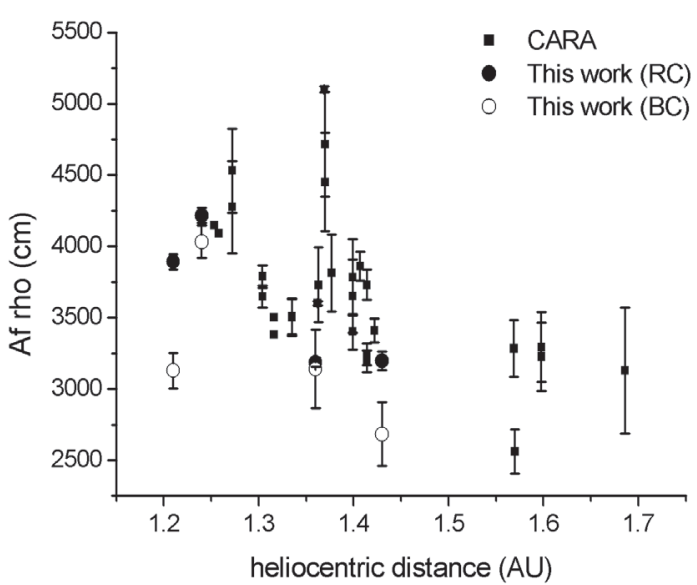

Fig. 1. The variation in Af $\rho$ (only the pre-perihelion points are plotted) with heliocentric distance. The square symbols are given by Sostero \& Ligustri $(2004,2005)$ in the Cometary Archive for Amateur Astronomers (CARA), respectively. The filled and open circles are determined from observations with $\mathrm{RC}$ and $\mathrm{BC}$ filters in this work.

\section{Dust properties and gas production rates}

To characterize the dust activity of a comet it has become customary to determine the product of albedo $(A)$, filling factor $(f)$, and the projected radius $(\rho)$ of the aperture in the plane of the comet used for observations, Af $\rho$ (A'Hearn et al. 1984), given by:

$A f \rho=\frac{(2 r \Delta)^{2}}{\rho} \frac{F_{\mathrm{com}}}{F_{\text {sun }}}$.

Here $r[\mathrm{AU}]$ is the comet's heliocentric distance, $\Delta[\mathrm{cm}]$ the comet's geocentric distance, $\rho[\mathrm{cm}]$ the radius of aperture at the comet $\left(20000 \mathrm{~km}\right.$ in this work), $F_{\text {com }}\left[\mathrm{erg} \mathrm{cm}^{-2} \mathrm{~s}^{-1}\right]$ 

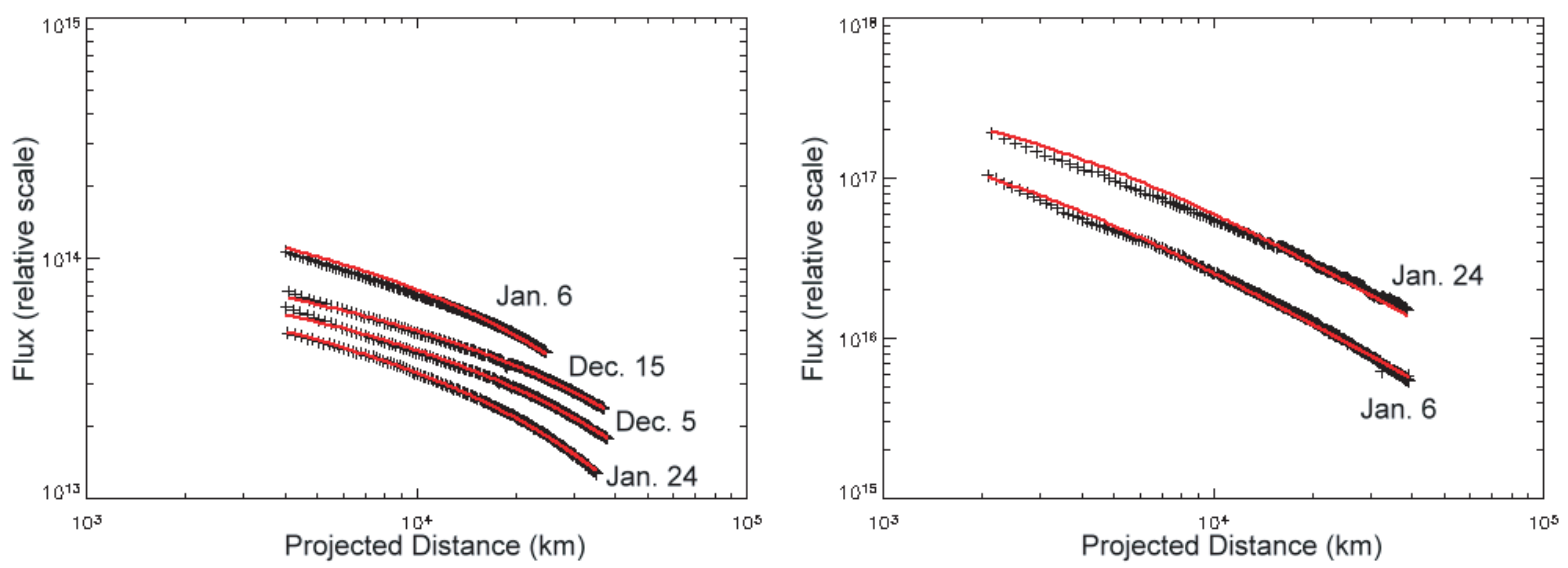

Fig. 2. Mean-radial column-density profiles of $\mathrm{C}_{2}$ (left panel) and $\mathrm{NH}_{2}$ (right panel). The best-fitting Haser profiles are shown by solid curves.

Table 4. Production rates of $\mathrm{C}_{2}$ and $\mathrm{NH}_{2}$ and scale lengths, $l_{\mathrm{p}}$ and $l_{\mathrm{d}}$, of parent and daughter molecules, respectively.

\begin{tabular}{lcccccc}
\hline \hline Date(UT) & $\begin{array}{c}Q\left(\mathrm{C}_{2}\right) \\
10^{26} \text { molecule s }^{-1}\end{array}$ & $\begin{array}{c}l_{\mathrm{p}}\left(\mathrm{C}_{2}\right) \\
10^{4} \mathrm{~km}\end{array}$ & $\begin{array}{c}l_{\mathrm{d}}\left(\mathrm{C}_{2}\right) \\
10^{5} \mathrm{~km}\end{array}$ & $\begin{array}{c}Q\left(\mathrm{NH}_{2}\right) \\
10^{26} \text { molecule s}^{-1}\end{array}$ & $\begin{array}{c}l_{\mathrm{p}}\left(\mathrm{NH}_{2}\right) \\
10^{3} \mathrm{~km}\end{array}$ & $\begin{array}{c}l_{\mathrm{d}}\left(\mathrm{NH}_{2}\right) \\
10^{5} \mathrm{~km}\end{array}$ \\
\hline Dec. 05 2004 & $1.07 \pm 0.19$ & $2.81 \pm 0.35$ & $6.71 \pm 0.05$ & & & \\
Dec. 15 2004 & $1.13 \pm 0.26$ & $4.50 \pm 0.22$ & $2.52 \pm 0.70$ & & & \\
Jan. 06 2005 & $1.38 \pm 0.19$ & $1.88 \pm 0.93$ & $3.84 \pm 0.84$ & $0.87 \pm 0.12$ & $2.12 \pm 0.41$ & $5.61 \pm 0.29$ \\
Jan. 24 2005 & $3.76 \pm 0.63$ & $3.03 \pm 0.42$ & $1.25 \pm 0.35$ & $1.03 \pm 0.17$ & $3.30 \pm 0.03$ & $5.30 \pm 1.50$ \\
\hline
\end{tabular}

the measured cometary flux in a continuum filter, and $F_{\text {sun }}\left[\mathrm{erg} \mathrm{cm}^{-2} \mathrm{~s}^{-1}\right]$ the solar flux at $1 \mathrm{AU}$. The solar flux was computed by using a high-resolution solar spectrum (Kurucz et al. 1984) for determining the solar flux within the wavelength range on which the transmission curve of the comet continuum filters is larger than zero. The $A f \rho$ is related to a stationary model that assumes a uniform and constant dust expansion within the coma. According to this model, the dust column density varies as $\rho^{-1}$. In this case, $A f \rho$ becomes independent of $\rho$. The derived values for $A f \rho$ on the four photometric nights are presented in Table 3. In Fig. 1, the $A f \rho$ values are plotted versus heliocentric distance, together with $A f \rho$ values taken from other publications.

\subsection{Dust colors}

The derived $A f \rho$ values in two filter bandpasses allow for the determination of the color of the cometary dust. The dust color is defined as the gradient of the $A f \rho$ product between the blue $\left(\lambda_{0}=445 \mathrm{~nm}\right)$ and red $\left(\lambda_{0}=687.4 \mathrm{~nm}\right)$ continuum points (Jewitt $\&$ Meech 1986). The color can be quantified in percentage reddening per $1000 \AA$, using the relation (Bonev 2002):

color $=\frac{A f \rho_{687}-A f \rho_{445}}{\lambda_{1}-\lambda_{2}} \frac{2000}{A f \rho_{687}+A f \rho_{445}}$,

where $A f \rho_{687}$ and $A f \rho_{445}$ are the $A f \rho$ values at wavelength $687.4 \mathrm{~nm}\left(\lambda_{1}\right)$ and $445 \mathrm{~nm}\left(\lambda_{2}\right)$, respectively. The resulting dust colors are presented in Table 3 . The average dust color is $4.7 \%$ per $1000 \AA$, which means that the dust coma of comet Machholz is redder than the solar spectrum.

\subsection{Gas production rates}

In order to determine the gas production rates, the continuum contribution in the gas filter images can be removed by the continuum scaling factor (Kursun et al. 2002). The scaling factor $k$

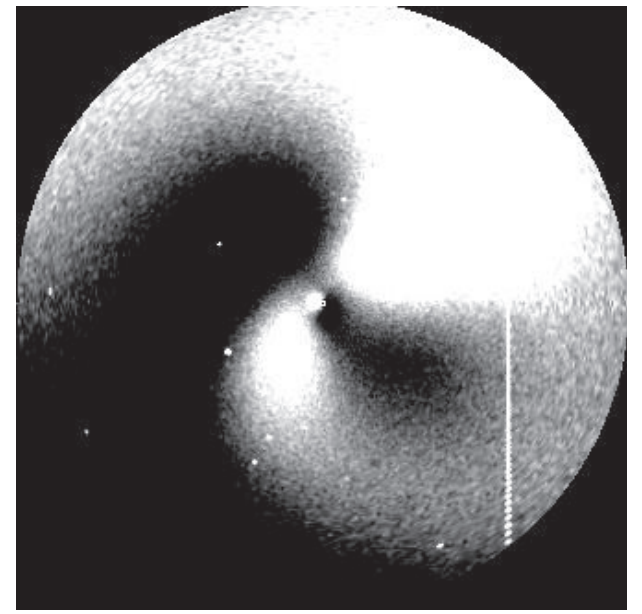

Fig. 3. An image of comet Mochholz taken with $\mathrm{CN}$ filter on 2004 Dec. 9 at La Silla Observatory, ESO. The image shows the deviation from the mean coma intensity profile and the gray-stone scale is set between 0.95 and 1.05; North is up, East to the left.

is given by

$k=\frac{F_{m+d}^{\odot}}{F_{d}^{\odot}}\left[1+\frac{A f \rho_{687}-A f \rho_{445}}{\lambda_{687}-\lambda_{445}} \frac{\lambda_{m+d}-\lambda_{d}}{(A f \rho)_{d}}\right]$.

Here the subscripts 687 and 445 refer to the red continuum and blue continuum filters and the subscripts $m+d$ and $d$ refer to gas emission filter and the dust continuum filter nearby (i.e. blue continuum for $\mathrm{C}_{2}$ and red continuum for $\mathrm{NH}_{2}$ ). $F^{\odot}$ denotes the solar flux convoluted with the transmission curve of the filter indicated in the subscript, $\lambda$ the wavelength.

After the continuum subtraction, the mean radial emission profiles of $\mathrm{C}_{2}$ and $\mathrm{NH}_{2}$ were derived from the images obtained in the corresponding filters. We fitted the mean radial brightness profiles of $\mathrm{C}_{2}$ and $\mathrm{NH}_{2}$ using the Haser model (Haser et al. 1957), which describes the isotropic emission of cometary 


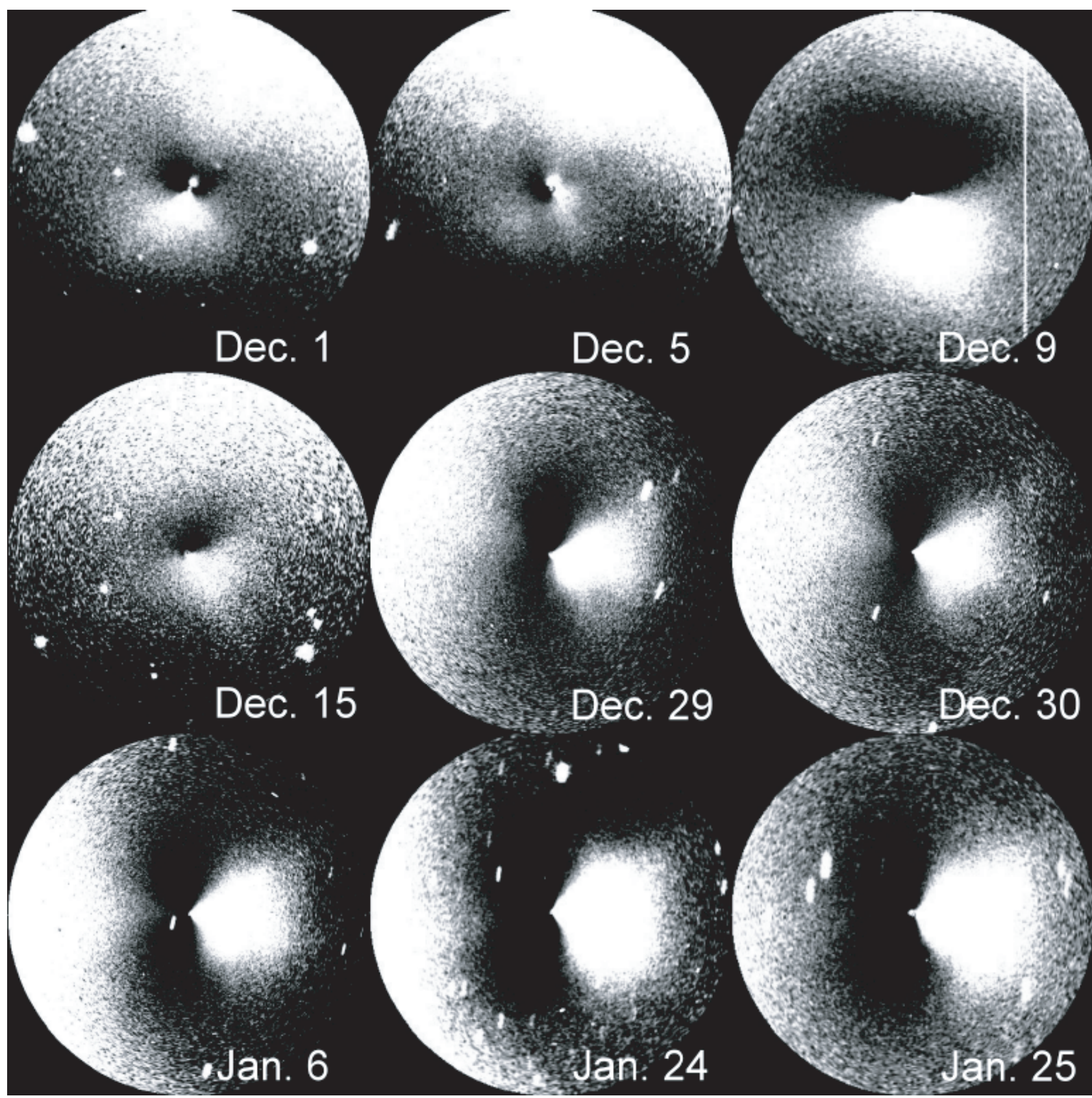

Fig. 4. Sequence of Red continuum images after coma structure enhancement. The nucleus position is at the center of each frame, and the diameter of field of view is $80000 \mathrm{~km}$. The brightness is scaled $\pm 5 \%$ around the mean instensity value. North is up, East to the left, and the arrows indicate the projected solar direction.

neutral molecules and their daughter molecules and radicals. The scale lengths of the parent molecules $\left(l_{\mathrm{p}}\right)$ and the daughter molecules $\left(l_{\mathrm{d}}\right)$ can be estimated from a fit of the model output to the observational data. These quantities determine the shape of the radial brightness profiles. The derived scale lengths are presented in Table 4 . When the brightness profiles are converted into column density profiles, we can then go on to compute the corresponding Haser parent production rates $(Q)$. For the conversion into column density profiles, the fluorescence efficiency factors ( $g$-factors) of $\left.\mathrm{C}_{2}\left(4.5 \times 10^{-13} \mathrm{erg} \text { (molecule s) }\right)^{-1}\right)$, A'Hearn et al. 1995) and $\mathrm{NH}_{2}\left(2.29 \times 10^{-15} \mathrm{erg} \text { (molecule s) }\right)^{-1}$, Korsun et al. 2002) were applied. In this calculations, we assumed that the $g$-factors varied with the heliocentric distance $r$ (AU) as $g=g_{0} / r^{2}$. The mean radial column density profiles derived from the observations during the four photometric nights are plotted in Fig. 2, together with the best-fitting profiles obtained with the Haser model. The production rates of the $\mathrm{C}_{2}$ and $\mathrm{NH}_{2}$ Haser parents are summarized in Table 4.

\section{Coma morphology and jet activity}

To study the morphology of the gas and dust coma of comet Machholz, an image enhancement technique was applied to the present set of images. The mean radial brightness profile of the cometary coma, averaged over all azimuthal angles, was determined in the first step. Then, the comet image was divided by the mean brightness profile to enhance deviations from the mean coma. This method was applied to all images taken in continuum filters, the $\mathrm{CN}$ filter, and the $\mathrm{C}_{2}$ filter (before continuum subtraction). Since the signal-to-noise ratio of the images taken 


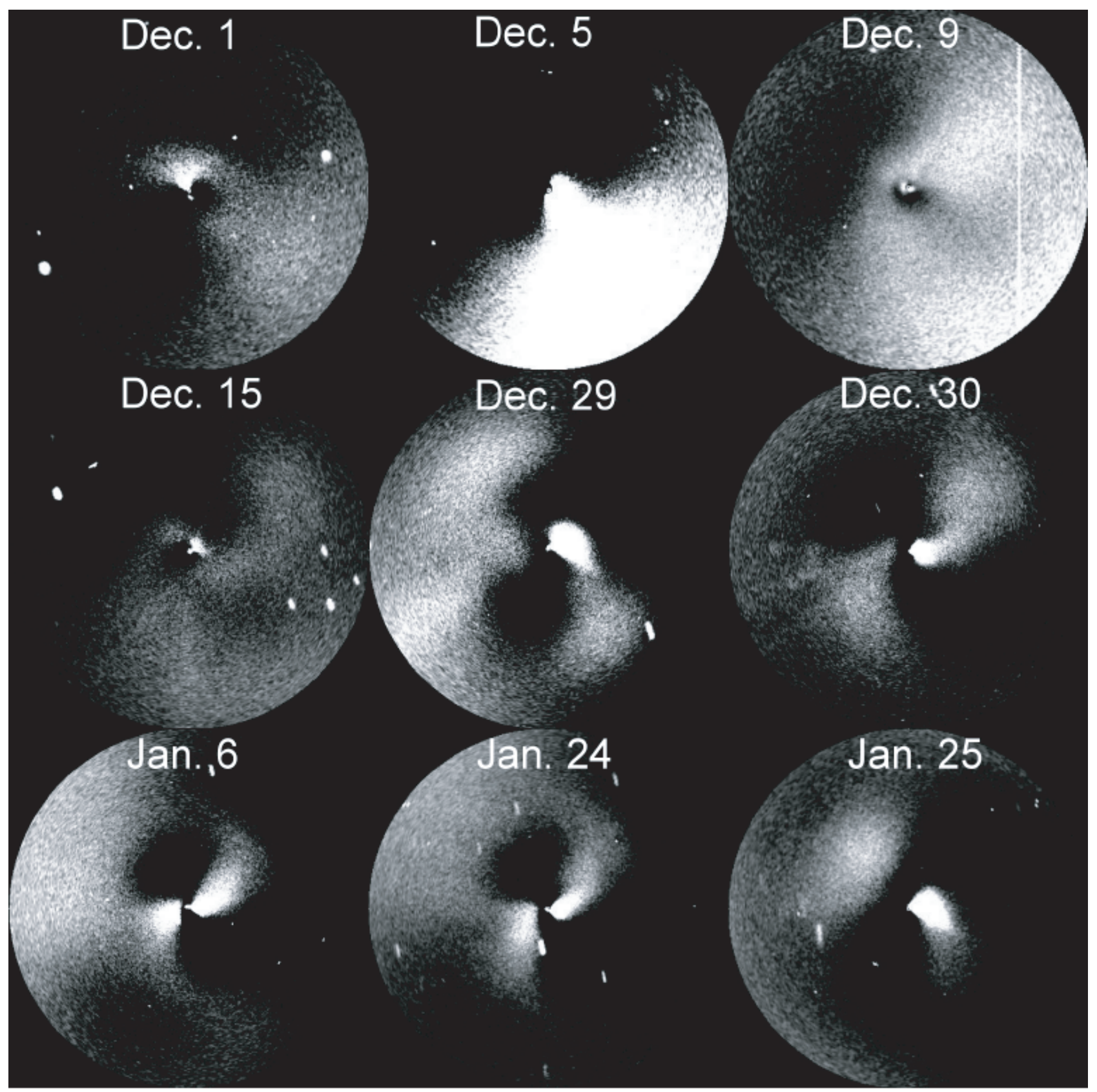

Fig. 5. As in Fig. 4, but for $\mathrm{C}_{2}$ filter images.

in the $\mathrm{NH}_{2}$ filters was low, these images are not suitable for an analysis of the coma morphology. The processed $\mathrm{C}_{2}$ images of the coma of comet Machholz are presented in Fig. 5. The processed $\mathrm{C}_{2}$ filter images from observations between December 9 and January 24 revealed two jets in the coma of comet Machholz. These jets appeared to be twisted into a spiral-like structure, and the position angles at which the jets are observed clearly changed between December 9 and December 30. Between January 6 and January 24 , no change in the position of the two jets was detectable. Between January 24 and January 25, the appearance of the $\mathrm{C}_{2}$ coma had changed. One jet has disconnected from the nucleus position and an isolated cloud remained in the coma, while the curvature of the second jet has changed. In Fig. 3, two twisted jets could also be revealed in the images taken in the $\mathrm{CN}$ filter on December 9. (In all images presented here, North is at the top and East is at the left.)
The processed images taken in the Red continuum filter are shown in Fig. 4. Here, the coma has a different appearance compared to the $\mathrm{C}_{2}$ images (Fig. 5). The two jets observed in the gas images cannot be detected in the continuum images. The dust coma shows an asymmetry with enhanced brightness in the projected solar direction.

\section{Discussion}

The observed jets in the gas coma can be explained by the presence of two active regions on the nucleus surface of comet Machholz. The rotation of the nucleus may cause the spiral shape of the jets and its change in position angle with time. Unfortunately, the time coverage of the images is insufficient for deriving the rotation period of the nucleus or the orienting of its rotation axis. The disconnection of one $\mathrm{C}_{2}$ jet observed between 
January 24 and January 25 can be explained if it is assumed that one active surface area has moved out of the sunlight and into the shadow of the nucleus. In such a case, the sublimation of ices from the surface area stops and the area can become inactive within a short time.

The enhancement in brightness in the dust coma is always directed towards the projected solar direction and not correlated to the position of the gas jets. Therefore, it is likely that dust jets and gas jets came from different sources. Organic "CHON" grains (Wickramasinghe \& Allen 1986; Kissel et al. 1986a,b) coming from active regions might be a source of $\mathrm{CN}$ and $\mathrm{C}_{2}$ radicals in the jets. These grains are probably not larger than $0.1 \mu \mathrm{m}$, so that they were not seen in the reflected continuum, which was dominated by much larger grains (Sekanina 1986). That also explains why the jets observed in the $\mathrm{C}_{2}$ filter images are weaker than in the $\mathrm{CN}$ filter images. The continuum provides a relatively larger contribution to the $\mathrm{C}_{2}$ filter images than to the $\mathrm{CN}$ filter images and itself did not show jet structures.

\section{Summary}

The summary of our observations of the comet Machholz were showed in the following:

1. For comet Machholz, the average values of the $\mathrm{C}_{2}$ parent and daughter scale lengths are $3.45 \times 10^{4} \mathrm{~km}$ and $3.49 \times$ $10^{5} \mathrm{~km}$, respectively. The corresponding values for $\mathrm{NH}_{2}$ are $2.17 \times 10^{3} \mathrm{~km}$ and $3.01 \times 10^{5} \mathrm{~km}$, respectively. In comparison, the presented values of the $\mathrm{C}_{2}$ parent and daughter scale lengths are close to our previous measurements $\left(1.8 \times 10^{4} \mathrm{~km}\right.$ and $\left.2.0 \times 10^{5} \mathrm{~km}\right)$ of comet $\mathrm{C} / 2001 \mathrm{~A} 2$ (LINEAR) (Lin et al. 2007). The $\mathrm{NH}_{2}$ parent and daughter scale lengths are comparable to the corresponding value for comet C/1999 J3(LINEAR) $\left(5.0 \times 10^{3} \mathrm{~km}\right.$ and $\left.1.0 \times 10^{5} \mathrm{~km}\right)$ measured by Kousun et al. (2002)

2. The average $\mathrm{C}_{2}$ and $\mathrm{NH}_{2}$ Haser parent production rates determined in four nights at heliocentric distances between $1.43 \mathrm{AU}$ and $1.21 \mathrm{AU}$ are $Q\left(\mathrm{C}_{2}\right) \sim 1.84 \times$ $10^{26}$ molecules s ${ }^{-1}$ and $Q\left(\mathrm{NH}_{2}\right) \sim 0.95 \times 10^{26}$ molecules s $^{-1}$, respectively. These values are nearly the same as comet C/2001 A2 (LINEAR) $\left(Q\left(\mathrm{C}_{2}\right) \sim 1.3 \times 10^{26}\right.$ molecules s $^{-1}$ and $Q\left(\mathrm{NH}_{2}\right) \sim 0.88 \times 10^{26}$ molecules s $\left.{ }^{-1}\right)$ but less than two orders of magnitude in comparison to those of comet Hale-Bopp (Rauer et al. 2003).
3. The average $A f \rho$ parameters at wavelengths of $\lambda_{0}=445 \mathrm{~nm}$ and $\lambda_{0}=687.4 \mathrm{~nm}$ are $3245 \mathrm{~cm}$ and $3623 \mathrm{~cm}$ on the same interval as heliocentric distances, respectively.

4. The average of dust color is $4.7 \%$ per $1000 \AA$ on the wavelength interval [ $445 \mathrm{~nm}, 687.4 \mathrm{~nm}]$.

5. A morphological analysis of gas coma revealed spiral jets in the $\mathrm{CN}$ and $\mathrm{C}_{2}$ images. These jets are not present in the continuum images. The observed coma structures can be explained by the presence of two active surface regions on a rotating nucleus. However, the dataset is not sufficient to derive the period of the comets rotation. The disconnection of one jet from the nucleus position on January 25 is suggestive of the moving of one active surface region away from the sunlit direction.

Acknowledgements. This work was based on observations obtained at Lulin Observatory whose staff and the assistants kindly help me to finish these observations. The research carried out was supported by NSC95-2111-M-008-006.

\section{References}

A’Hearn, M. F., Schleicher, D. G., Feldmann, P. D., Millis, R. L., \& Thompson, D. T. 1984, AJ, 89579

A'Hearn, M. F., Hoban, S., Birch, P. V., et al. 1986, Nature, 327, 649

A'Hearn, M. F., Millis, R., Schleicher, D. G., Osip, D. J., \& Birch, P. V. 1995, Icarus, 118, 223

Bockelee-Morva, D., Crovisier, J., Mumma, M. J., \& Weaver, H. A. 2004, in Comet II, ed. M. C. Festou, H. U. Keller, \& H. A. Weaver (Tucson: University of Arizona Press), 745, 449

Bonev, T., Jockers, K., Petrova, E., et al. 2002, Icarus, 160, 419

Haser, L. 1957, Bull. Acad. R. Sci. Leige, 43, 740

Jewitt, D., \& Meech, K. J. 1986, ApJ, 310, 937

Kawakita, H., Watanabe, J., \& Kinoshita, D. 2001, ApJ, 572, L177

Kinoshita, D., Huang, K. Y., Wu, Y. L., Chang, Y. H., \& Urata, Y. 2003, Lulin Observatory Report, 30

Kinoshita, D., Chen, C. W., Lin, H. C., et al. 2005, CHJAA, 5, L315

Kissel, J., Brownlee, D. E., Buchler, K., et al. 1986a, Nature, 321, 280

Kissel, J., Sagdeev, R. Z., Bertaux, J. L., et al. 1986b, Nature, 321, 336

Kurucz, R. L., Furenlid, I., Brault, J., \& Testerman, L. 1984, Solar flux atlas from 296 to $1300 \mathrm{~nm}$ (Sunspot, New Mexico: National Solar Observatory)

Korsun, P. P., \& Jockers, K. 2002, A\&A, 381, L703

Lara, L.-M., Tozzi, G. P., Boehnhardt, H., DiMartino, M., \& Schulz, R. 2004, A\&A, 422, 717

Lin, Z. Y., Chang, C. P., \& Ip, W. H. 2007, AJ, 133, L1861

Rauer, H., Helbert, J., Arpigny, C., et al. 2003, A\&A, 397, 1109

Sastri, J. H., Vasundhara, R., Kuppuswamy, K., \& Velu, C. 2005, IAUC., 8480

Sekanina, Z. 1986, Adv. Space Res., 5, 307

Wickramasinghe, D. T., \& Aleen, D. A. 1986, Nature, 323, 44 